\title{
Grasp planning for a reconfigurable parallel robot with an underactuated arm structure
}

\author{
M. Riedel, M. Nefzi, and B. Corves \\ Department of Mechanism Theory and Dynamics of Machines (IGM), \\ RWTH Aachen University, Aachen, Germany
}

Received: 26 February 2010 - Revised: 22 June 2010 - Accepted: 19 August 2010 - Published: 22 December 2010

\begin{abstract}
In this paper, a novel approach of grasp planning is applied to find out the appropriate grasp points for a reconfigurable parallel robot called PARAGRIP (Parallel Gripping). This new handling system is able to manipulate objects in the six-dimensional Cartesian space by several robotic arms using only six actuated joints. After grasping, the contact elements at the end of the underactuated arm mechanisms are connected to the object which forms a closed loop mechanism similar to the architecture of parallel manipulators. As the mounting and grasp points of the arms can easily be changed, the manipulator can be reconfigured to match the user's preferences and needs. This paper raises the question, how and where these grasp points are to be placed on the object to perform well for a certain manipulation task.
\end{abstract}

This paper was presented at the IFToMM/ASME International Workshop on Underactuated Grasping (UG2010), 19 August 2010, Montréal, Canada.

\section{Introduction}

In recent years, manufacturing industry is governed by fundamental changes with regard to the conditions, like progressive globalization and rapid technological development as well as changes in the resources situation (Möller, 2008; Müller et al., 2010). Classical demands on handling systems are currently undergoing change. In the past higher load capacity, greater precision and higher speeds were the main demands in the present situation priorities are increasingly shifting towards customized production and flexible solutions to component dependent problems. Currently available handling systems are not completely efficient to fulfil the increased demands (Nyhuis, 2008) and one of the major problems is the automated handling of large components in small production series such as aerospace systems, shipbuilding, wind turbine construction or manufacture of solar panels etc.

A single robot with one large customized Gripper is not usually capable of moving the spacious component without subjecting it to any forces.

Correspondence to: $\mathrm{M}$. Riedel

(riedel@igm.rwth-aachen.de)
The concept of handling with co-operating robots represents a more versatile approach as the component can be gripped and supported at different points by several robots depending on the shape of the object (Feldmann et al., 2007). One disadvantage of having many industrial robots handling one part is the high overall number of actuators, e.g. 18 actuators for 3 robots to perform a 6 DOF object motion. The additional drives lead to a high redundant system which results in complex control architecture and high costs.

A novel reconfigurable handling system is presented in (Nefzi et al., 2006; Müller et al., 2010) and shown in Fig. 1. The system "PARAGRIP" provides the same versatility and flexibility as co-operating robots but at significant lower procurement and operating costs. It is based on a concept with a different actuator configuration featuring simpler and less number of drives. Due to this drive architecture the separate arms are underactuated and there is need that these arms should work together in combination. The contact element which connects the object with the arm is mounted on a free movable, spring centered wrist joint. This joint needs to be aligned passively when the contact element touches the surface of the object.

This paper deals with the grasping behavior and the optimization of grasp positions for the underactuated arms. 


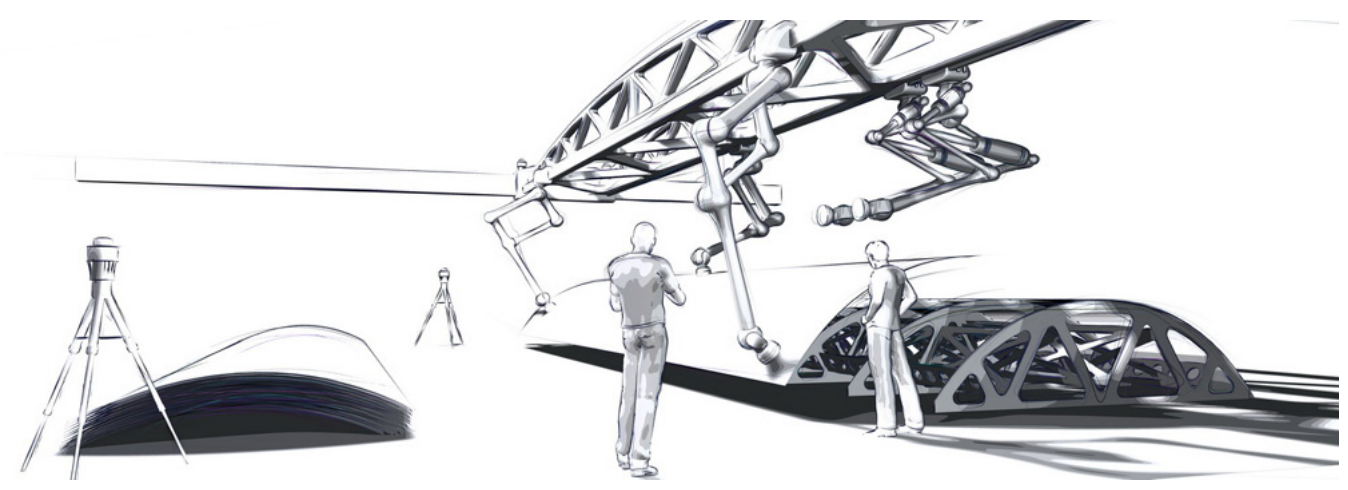

Figure 1. Design concept of the adaptable and reconfigurable handling system PARAGRIP.

Firstly we present the novel handling idea and the structure of the robot arms. Then we proceed towards the grasping process of the underactuated arms. Finally, we will present an approach for finding the optimal grasp points for a given object motion.

\section{Handling concept}

The aim of the new handling concept is to increase the flexibility and the automation in the assembly processes of large components in small series (Müller et al., 2010). This can be achieved by using a modular and adaptable assembly system which enables reconfiguration of the kinematic layout depending on the task. The main idea of this handling principle is based upon autonomous gripping and moving objects by using multiple arms within a parallel kinematic structure which is shown in Fig. 2. Whenever the grasping is done, the parallel kinematic structure is regenerated and integrates the object within the robot structure as a moveable platform. In contrast to mechanical handling systems that mimic human hands (Bicchi, 2000; Yoshikawa and Nagei, 1991) the object is not clamped or clutched to make a manipulation possible. The contact element at the end of each arm provides a temporary stable connection which can transfer forces and moments in all directions. The resulting closed-loop mechanism is formed by the arms and the object.

A complete manipulation sequence is schematically shown in Fig. 3. Here the object becomes part of the robot when the contact areas of the grippers are located on the object by the separate arms. Adhesion forces are generated by electro-magnets, vacuum cups, etc to ensure that the kinematic chains remain closed. This newly generated kinematic structure is similar to the structure of parallel robots. In contrast to cooperating robots, the configuration of the architecture in each object pose is determined by only six drives. After the motion the object is released and the connection between the arms is cut.

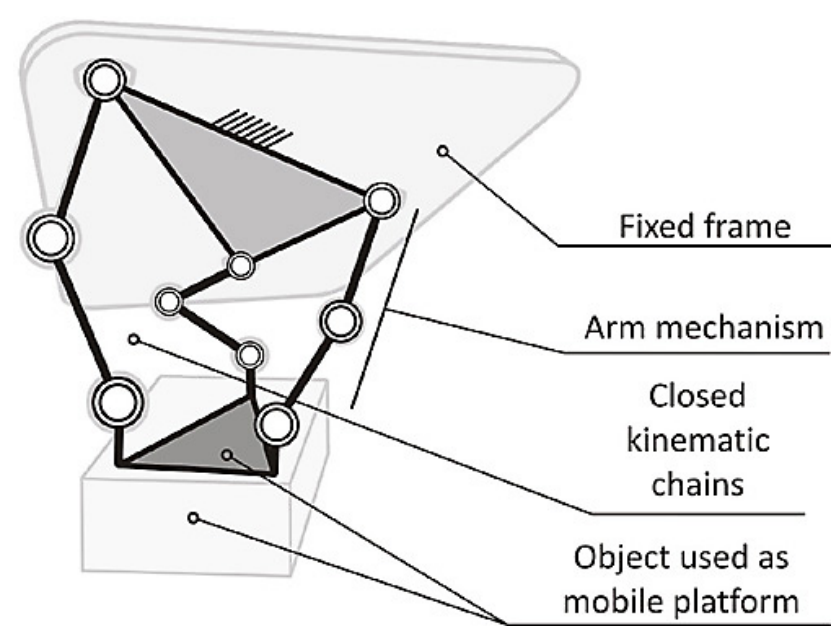

Figure 2. New handling principle.

\section{Robot architecture}

To perform a 6 DOF object motion with the prescribed handling concept a mobility number of six for each arm is necessary independently from the number of arms that are in contact with the object. A favorable configuration consists of three arms with six main actuators and three additional smaller actuators for grasping. During manipulation phase the additional drives can be disconnected, driven passively or support actively the object motion. As shown in Fig. 4 each arm mechanism is a combination of a five-bar-linkage with a parallelogram arrangement, a revolute joint around the vertical axis and a spherical wrist joint, similar to the structure presented in (Ebert-Uphoff I. and Gosselin, 1998).

Figure 5 illustrates the architecture of the wrist. It consists of two nested links (W1, W2) and one link (W3), which supports a contact element. Link 2 is the distal part of the arm mechanism. The axes of the three revolute joints (R, S and $\mathrm{T})$ intersect at one center of rotation.

There are two wrist configurations: in the first configuration all joints are passive and in the second one the joint $\mathrm{R}$ is 


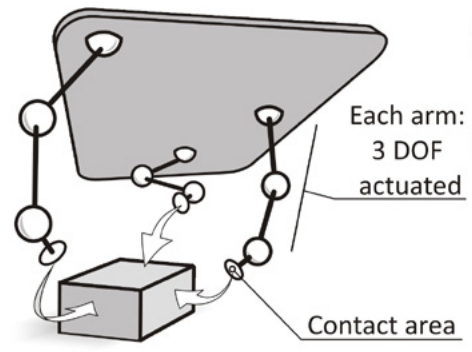

Phase 1: gripping

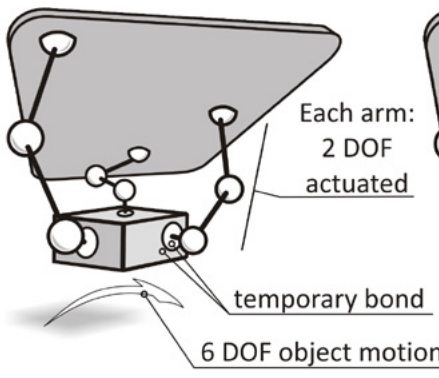

Phase 2: manipulation

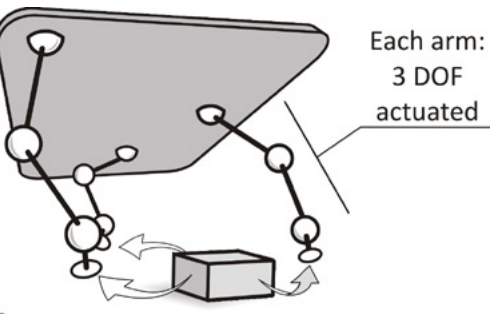

Phase 3: releasing

Figure 3. Movement sequence when handling an object.

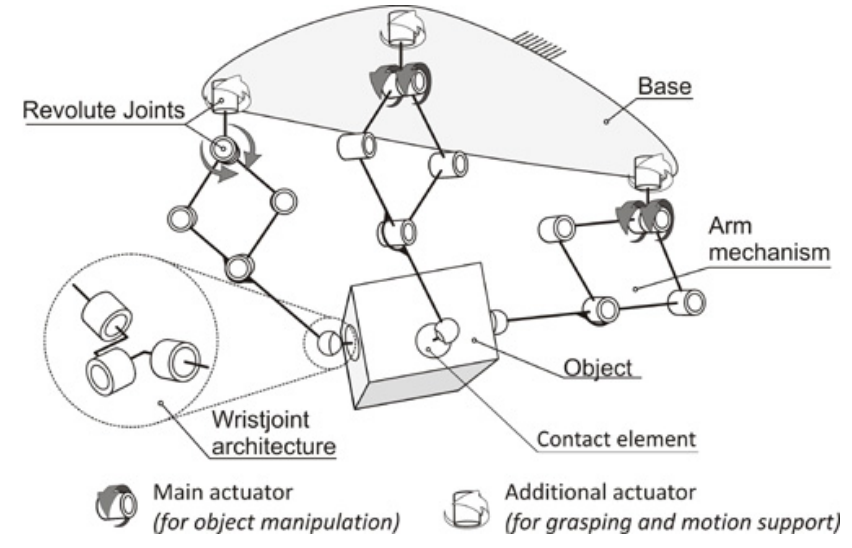

Figure 4. Robot structure.

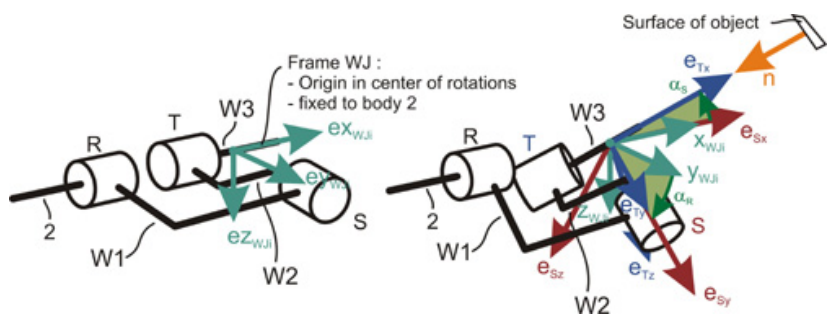

Figure 5. Wrist joint structure with frames and denotations. Left: center joint position. Right: general joint position.

driven by a low-cost stepper motor for grasping. The wrist is underactuated, which means all passive joints should be spring-centered to avoid collisions and grasp failure caused by undefined link positions. To reduce the necessary spring torque the common center of gravity of both links W2 and W3 together, is located at the center of rotation. The centering torque of joint $\mathrm{S}$ can be adjusted by tensioning two rotary springs.

The main drawback of this architecture is a singular joint configuration in center position, when axis $\mathrm{R}$ and $\mathrm{T}$ are collinear, see left part of Fig. 5. In this situation a rotation of link W3 about an axis perpendicular to $\mathrm{R}$ and $\mathrm{S}$ is not pos-

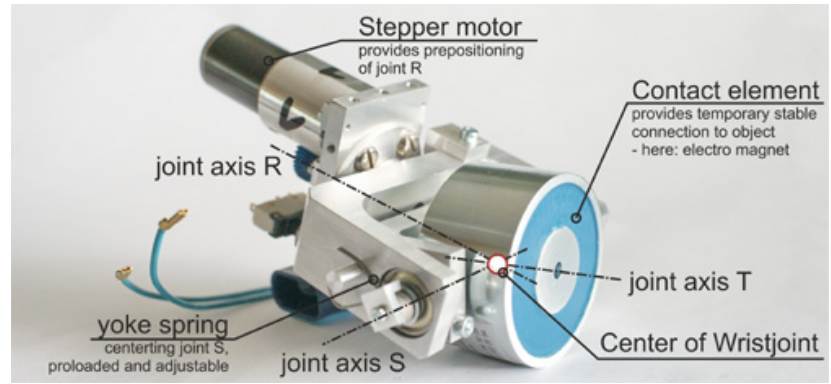

Figure 6. Wrist joint prototype.

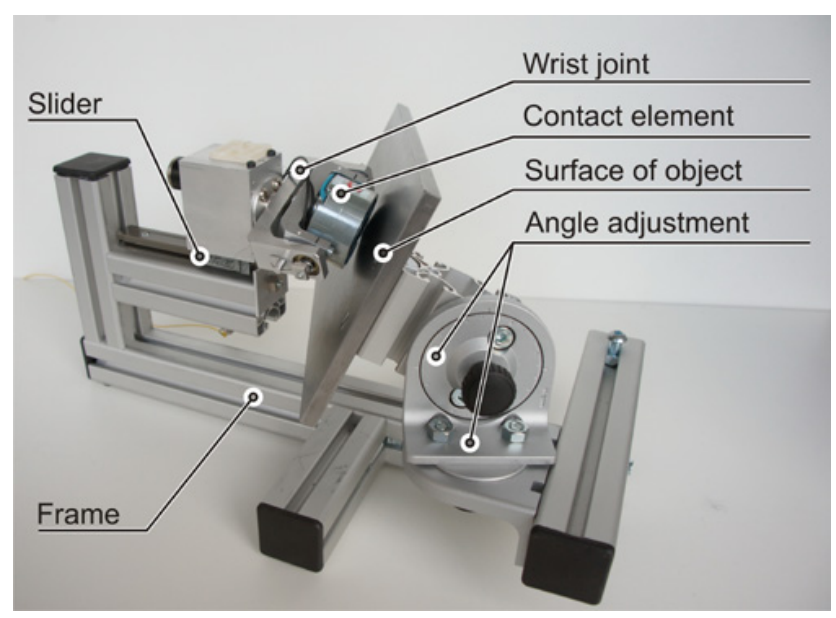

Figure 7. Wrist joint test bench.

sible. This aspect has a major effect on grasping behavior and needs to be carefully considered in motion- and grasp planning. Nevertheless, this wrist architecture fits best our requirements, which provides a wide range of motion. Rotation angle of $\pm 90^{\circ}$ in each axis is possible, in comparison to ball- or universal joints with $\pm 15^{\circ}$ and $\pm 45^{\circ}$ respectively. Furthermore, the chosen wrist joint has a short distance between the center of rotation and the surface of the contact element which enhances the passive grasp behavior. A picture of the above discussed wrist prototype including the electromagnet is shown in Fig. 6. 


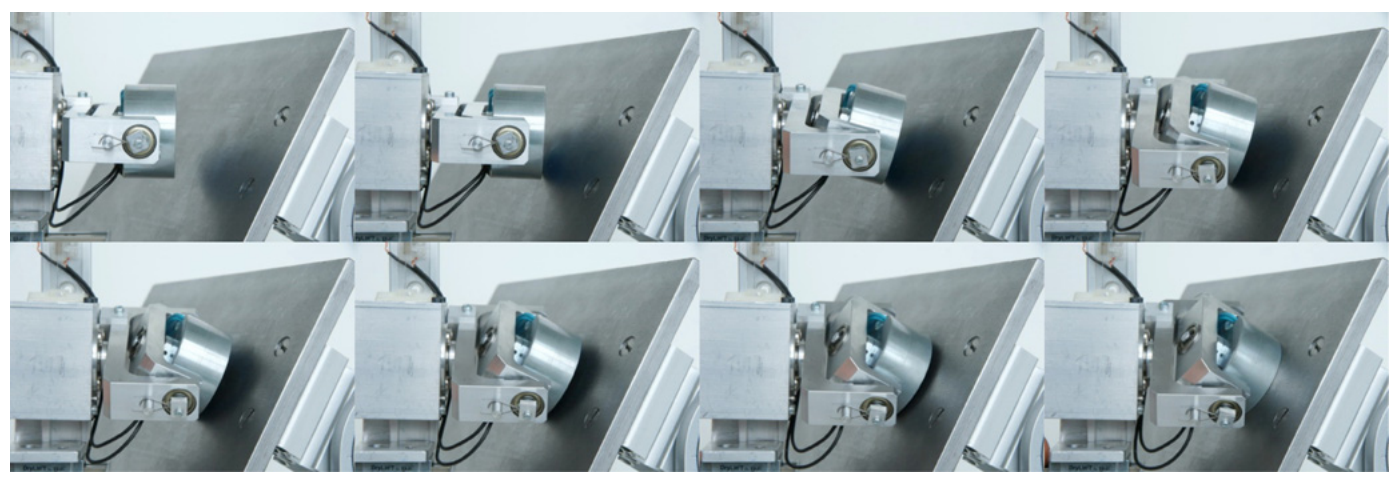

Figure 8. Wrist joint passive alignment.
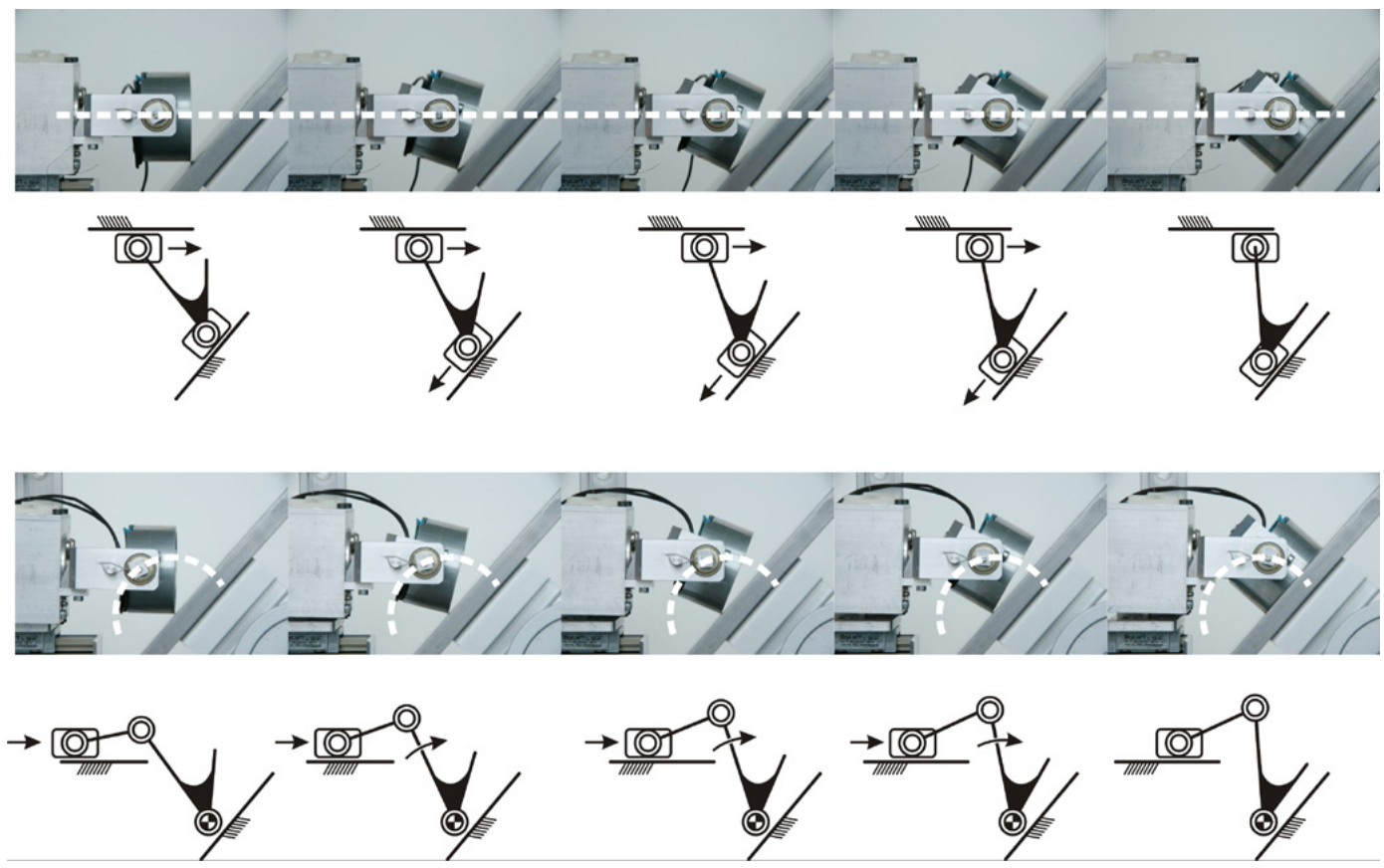

Figure 9. Comparison of linear and circular grasp motion.

\section{Grasp process}

One of the main advantages of this novel handling concept is its cost-effective build-up due to the reduced number of actuators. As explained in the previous sections the $3 \mathrm{DOF}$ wrist joint can be designed which free of drives. Hence, during grasping phase this underactuated structure needs to be driven by the external contact forces to ensure a correct alignment of contact element to the surface of the object which is similar to movement of the phalanx at a grasp process with an underactuated multi-finger gripper investigated in (Laliberté et al., 2002; Krut, 2005). This passive alignment depends here on several factors like the joint angles, friction conditions, grasp motion etc.

To calculate the necessary joint angles $\alpha_{\mathrm{R}}$ and $\alpha_{\mathrm{S}}$ for grasping, the orientations of the systems of coordinates $S$ and
T need to be determined, see Fig. 5. System $\mathrm{S}$ is attached to link W1, T to W3. Unit vector $\boldsymbol{e}_{\mathrm{Sy}}$ is collinear to joint axis $\mathrm{S}$, vector $\boldsymbol{e}_{\mathrm{Tx}}$ is in the direction of joint axis T. Vector $\boldsymbol{n}$ is normal to the surface. The vectors can be computed as follows:

$\boldsymbol{e}_{\mathrm{Sx}}=\boldsymbol{e}_{\mathrm{W}_{\mathrm{Jx}}}, \quad \boldsymbol{e}_{\mathrm{Sy}}=\frac{n \times \boldsymbol{e}_{\mathrm{W}_{\mathrm{Jx}}}}{\left\|n \times \boldsymbol{e}_{\mathrm{W}_{\mathrm{Jx}}}\right\|}, \quad \boldsymbol{e}_{\mathrm{Sz}}=\boldsymbol{e}_{\mathrm{Sx}} \times \boldsymbol{e}_{\mathrm{Sy}}$

$\boldsymbol{e}_{\mathrm{Tx}}=-\boldsymbol{n}, \quad \boldsymbol{e}_{\mathrm{Ty}}=\boldsymbol{e}_{\mathrm{Sy}}, \quad \boldsymbol{e}_{\mathrm{Tz}}=\boldsymbol{e}_{\mathrm{Tx}} \times \boldsymbol{e}_{\mathrm{Ty}}$

The joint angle $\alpha_{r}$ is the angle between the vectors $\boldsymbol{e}_{\mathrm{WJy}}$ and $\boldsymbol{e}_{\mathrm{Sy}}$ in direction of vector $\boldsymbol{e}_{\mathrm{WJx}}, \alpha_{s}$ between $\boldsymbol{e}_{\mathrm{Sx}}$ and $\boldsymbol{e}_{\mathrm{Tx}}$ in direction of $\boldsymbol{e}_{\text {Sy. }}$.

To investigate the grasp behavior a test bench has been developed which is shown in Fig. 7. The wrist joint prototype is located on a slider and can be moved towards a test surface. This surface is adjustable in every combination of the angles 

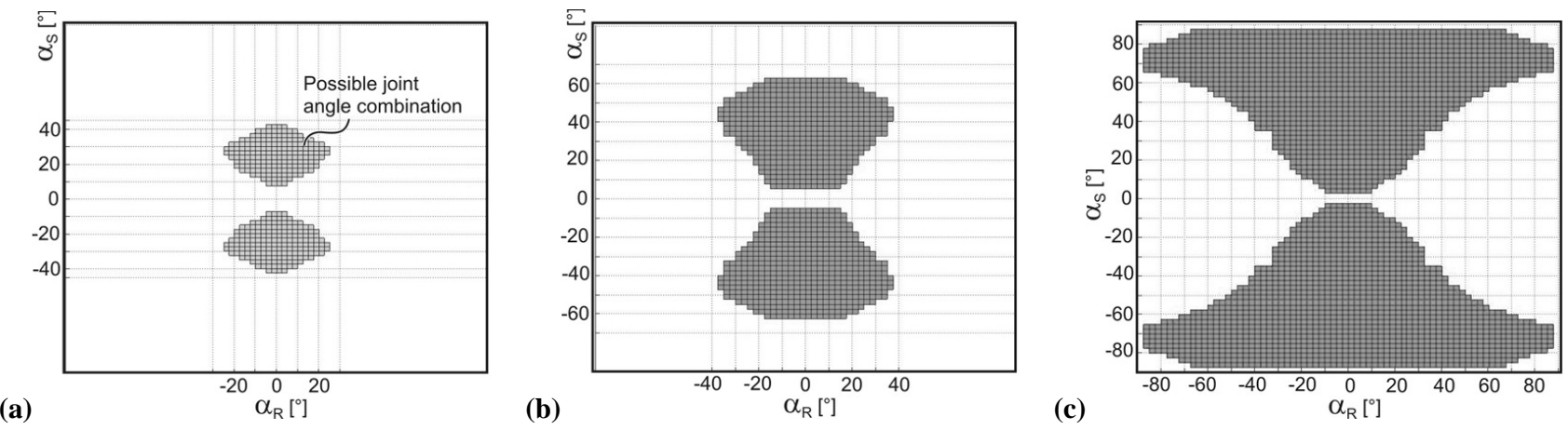

Figure 10. Successful passive alignment of the contact element for (a) linear motion $(\mu=0.2)$, circular motion $(\mu=0.2)(\mathbf{b})$ and circular motion and contact modification $(\mu=0.6)$ (c) while grasping.

$\alpha_{\mathrm{R}}$ and $\alpha_{\mathrm{S}}$. The test bench can be configured in many variations to test different grasping conditions by changing grasp paths, friction conditions etc. Figure 8 shows a sequence of a grasping procedure, where the contact element aligns passively to the test surface by rotating about the both angles $\alpha_{\mathrm{R}}$ and $\alpha_{\mathrm{S}}$.

A change in the configuration of the test bench enables the guidance of the joint on different paths. Figure 9a shows a linear grasp motion, when the center of the wrist joint is guided on a straight line. Here the contact point needs to slide on the surface which is similar to a double-slider-mechanism. At circular motion the contact element rolls on the fixed contact point by moving the center of the wrist joint on a radius like a reversed slider-crank-mechanism as shown in Fig. 9b.

The test results show that there is a significant difference in the passive alignment of the contact element for these grasping paths. It can be shown, that independent from friction conditions the circular grasp motion is always more successful. Successful grasping means that the contact element is correctly attached to the surface of the object without any misalignment.

An additional enhancement can be achieved by modifying the contact conditions using supplementary friction elements like rubber rings at the edge of the contact element.

Figure 10 shows the area of a successful passive alignment in the space of the wrist joint angles $\alpha_{\mathrm{R}}$ and $\alpha_{\mathrm{S}}$ for friction coefficient $\mu=0.2$ respectively $\mu=0.6$ at contact point and different grasp motions.

To understand the grasp behavior Fig. 11 depicts the contact situation for an arbitrary joint angle $\alpha_{\mathrm{S}}$ and the center position of $\alpha_{\mathrm{R}}$ when the edge of the contact element first hits the object.

When hitting the object a contact force $F_{\mathrm{K}}$ arises from Point $\mathrm{K}$ in the direction of the center of the wrist joint $C_{\mathrm{WJ}}$. If this force is inside the friction cone, normal and frictional forces $\left(F_{\mathrm{N}}\right.$ and $\left.F_{\mathrm{R}}\right)$ can be transferred from the surface to the contact element. Otherwise the maximum possible frictional force $\left(F_{\mathrm{R} 1}\right)$ is lower than the required frictional force $\left(F_{\mathrm{R} 1}+F_{\mathrm{R} 2}\right)$ in the contact point in such a way as no static

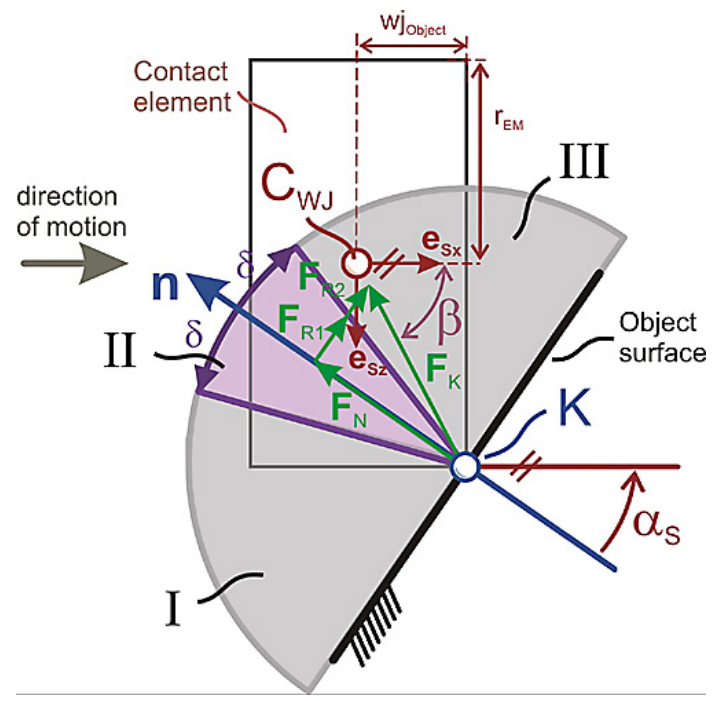

Figure 11. Force condition at contact point while grasping.

equilibrium can be found. Consequently the contact point has to slip away. As grasping with a linear motion requires a sliding of the contact point, the center of the wrist joint $C_{\mathrm{WJ}}$ needs to be located in the area III to succeed grasping. A force direction in area II causes jamming and the object will be displaced unintended. Here grasping is not successful. For circular motion both areas II and III are feasible. If the contact force shows in direction of area I the contact element will slip away in the wrong direction in both motion cases.

The maximum possible joint angle $\alpha_{\mathrm{S} \text { max }}$ for a successful grasping is computed for $\alpha_{\mathrm{R}}=0^{\circ}$ in the following way:

$\delta-\arctan (\mu), \beta=\arctan \left(\frac{r_{\mathrm{FM}}}{w j_{\mathrm{offset}}}\right)$

Linear grasp motion: $\quad \alpha_{\mathrm{S} \max }=\beta-\delta=43.1^{\circ}$ for $\mu=0.2$

Circular grasp motion: $\alpha_{\mathrm{S} \max }=\beta+\delta=65.7^{\circ}$ for $\mu=0.2$

$$
\alpha_{\mathrm{S} \max }=85.4^{\circ} \quad \text { for } \mu=0.6
$$


(a)

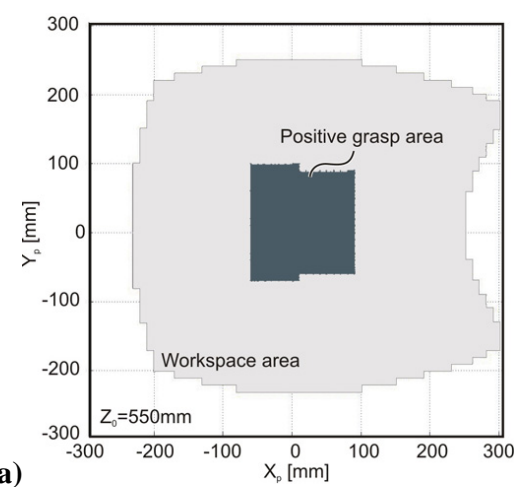

(b)

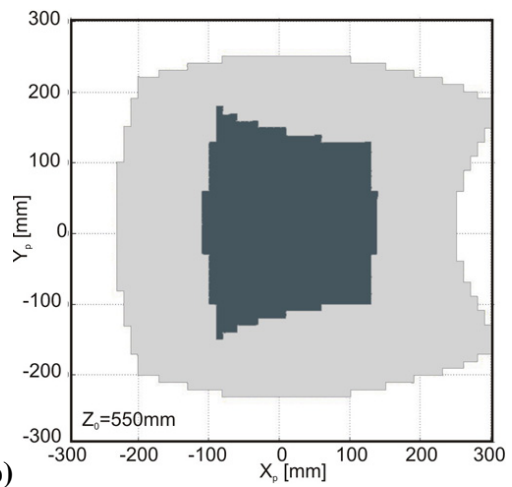

(c)

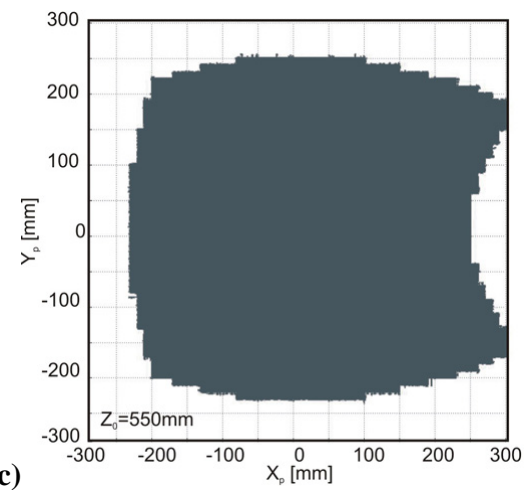

Figure 12. Successful grasp- and workspace area for linear motion (a), circular motion (b) and circular motion with contact element modifications (c).

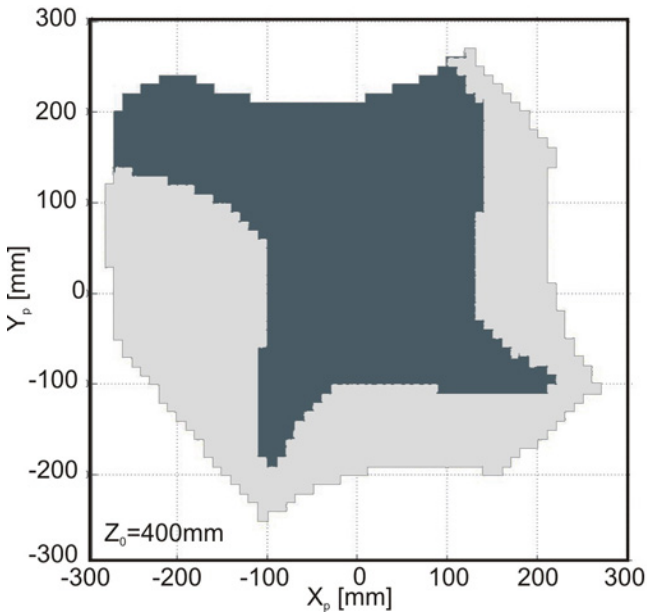

Figure 13. Grasping behavior for object orientation $\phi=45^{\circ}$ and wrist joint configuration I.

These angle limits can also be seen well in the experimental results. The equations also show that independent from friction conditions a circular grasp motion is always better. Above all higher friction at contact points affects the passive alignment at the circular motion in a positive way and at the linear motion in a negative way.

Investigation of positive grasp behavior in Cartesian space is more interesting than in joint space. Figure 12 shows the workspace for object manipulation in a single layer (light grey part) and the area (dark grey) where a cube with an edge length of $150 \mathrm{~mm}$ can be grasped. Analogous to the case of Fig. 10, Fig. 12a shows the result for linear motion, Fig. 12b for circular motion and Fig. 12c for a circular motion with a modified contact element. In the last case the higher friction causes a very good grasp behavior so that the object can be picked up all over the workspace. Investigations with different object orientations on the ground show that even with a circular grasp motion and an enhanced contact element some object poses at the edges of the workspace area cannot be

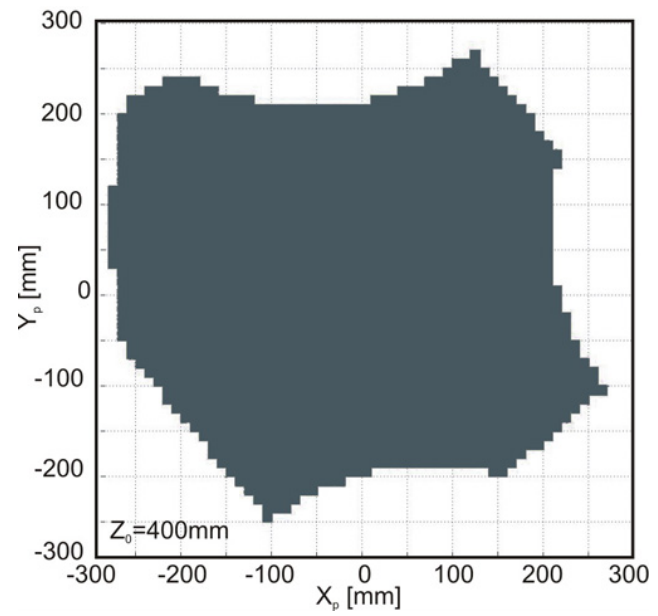

Figure 14. Enhanced grasping with object orientation $\phi=45^{\circ}$ and wrist joint configuration II.

grasped because of an improper combination of the joint angles $\alpha_{\mathrm{R}}$ and $\alpha_{\mathrm{S}}$, see Fig. 13. This drawback can be avoided by using the before mentioned wrist joint configuration II with an actuator in joint axis $\mathrm{R}$. This actuator is a very small, low cost stepper drive, which controls actively the position of the angle $\alpha_{\mathrm{R}}$ before or while grasping. During object manipulation the stepper is driven passively by the close loop kinematic chains of the complete robot architecture.

Figure 14 shows that a prepositioning of joint angle $\alpha_{\mathrm{R}}$ enables to pick up the object in any pose on this workspace layer.

To sum up the tests show that a positive passive alignment of the contact element can be achieved for any object pose on ground level by the three modifications depicted in Fig. 15:

- Guiding the wrist joint center on a circle

- Enhancing the frictional conditions at contact point by modifying the contact element

- Prepositioning of joint angle $\alpha_{\mathrm{R}}$ by a low cost stepper 


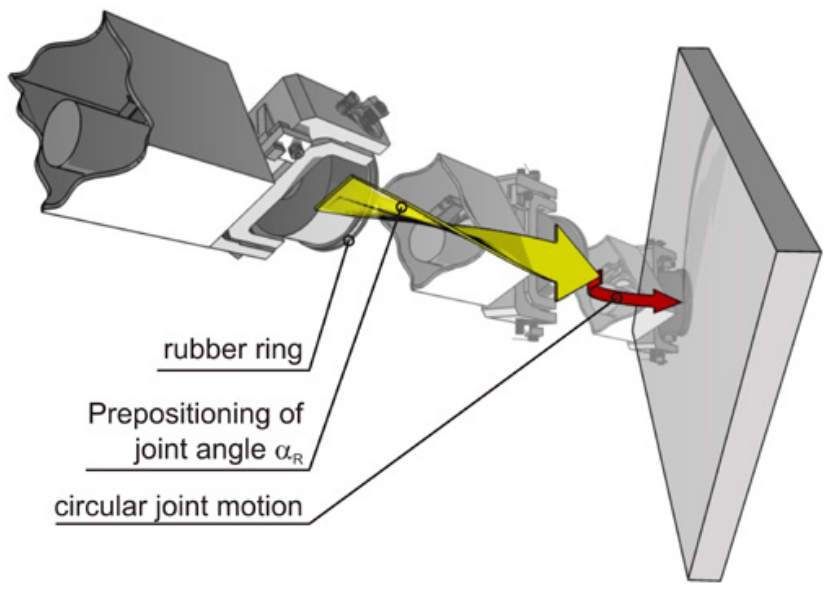

Figure 15. Enhancements for successful grasping

The friction conditions can be enhanced by using a ring of rubber on the edge of the contact element. The necessary joint angle $\alpha_{\mathrm{R}}$ for grasping can be computed with the help of Eq. (1) and is used for prepositioning.

Finally the exact circular path in 3-D space needs to be determined to achieve optimal grasp behavior. This can be done with the vectors starting from origin $O_{w j}$ which are shown in Fig. 16.

$\boldsymbol{b}_{\mathrm{S}}=-\boldsymbol{e}_{n} \cdot w j_{\mathrm{offset}}+\boldsymbol{e}_{\mathrm{TZ}} \cdot r_{\mathrm{EM}}-\boldsymbol{e}_{\mathrm{SZ}} \cdot r_{\mathrm{EM}}-\boldsymbol{e}_{\mathrm{SX}} \cdot w j_{\mathrm{offset}}$

$\boldsymbol{b}_{\mathrm{M}}=-\boldsymbol{e}_{n} \cdot w j_{\mathrm{offset}}+\boldsymbol{e}_{\mathrm{TZ}} \cdot r_{\mathrm{EM}}$

where $w j_{\text {Object }}$ is the distance between the joint center and the contact surface and $r_{\mathrm{EM}}$ is the radius of the contact element. The computed complete grasp paths of the wrist joint centers for all three arms are shown in Fig. 17. The first arbitrary part, here is a straight motion, and the second part is the circle which may have different curvatures at transition. This requires to be considered in trajectory planning to avoid vibrations.

\section{Grasp point optimization}

As the handling concept is based on the integration of the object into the kinematic structure, some robot performances can be directly affected by choosing adequate positions of the connecting points while grasping. The influence of a bad choice of these grasp points becomes very clear when taking a look at the following two examples. If the grasp points lie at or close to an imaginary line the complete robot configuration becomes uncertain in every object pose. In this singular situation the robot structure cannot withstand a wrench on the object and has a possibility to collapse. In a different case the grasp points are positioned well for force transmission but may reduce the mobility of the object, as for example large rotations could cause collisions.

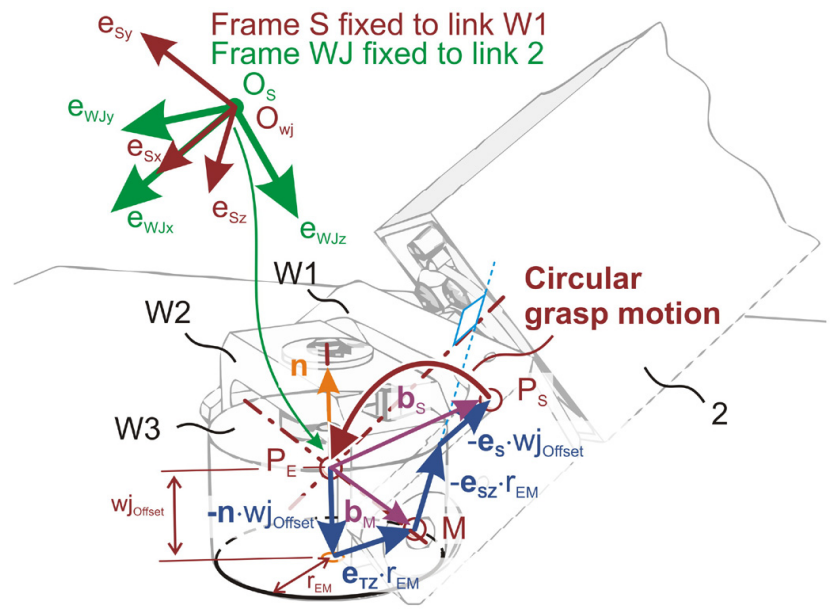

Figure 16. Computation of the circular grasp motion.

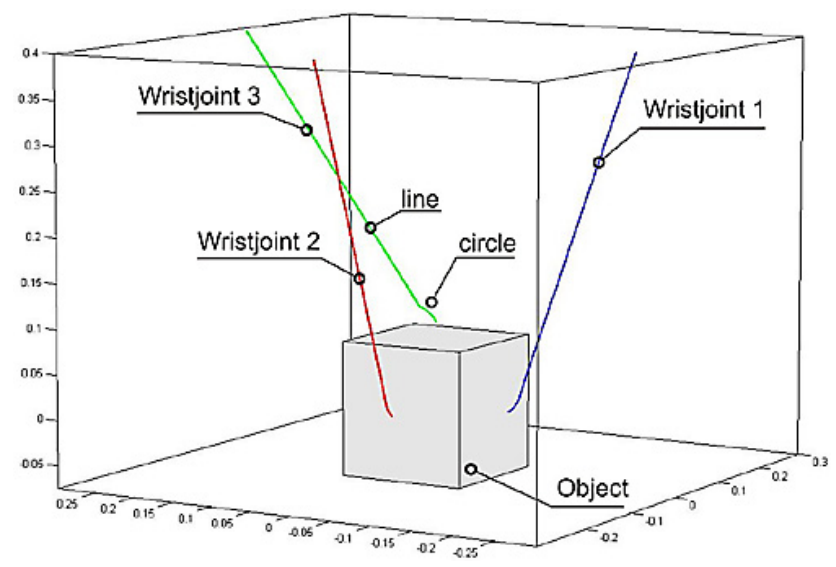

Figure 17. Visualization of the computed grasp paths.

That means by choosing the connection points some kinematic dimensions can be designed depending on the task to enhance workspace and dexterity, force- and velocity transmission along with accuracy and stiffness performance. As the selection of an appropriate grasp point combination is very challenging to a normal user an automatic optimization strategy is required.

A feasible optimization sequence which supports the user when planning the robot motion is shown in Fig. 18.

Among the information about the manipulation task, the optimization aim and the restrictions especially the possible grasp positions on the object are of importance. Therefore these positions could be generated automatically by scanning CAD data or be can be inputted manually by the user. The optimization algorithms can handle both, discrete and continuous descriptions of possible grasp areas, like a list of coordinates or a set of boundary for given surfaces. After computing an appropriate configuration the object motion can be performed. 


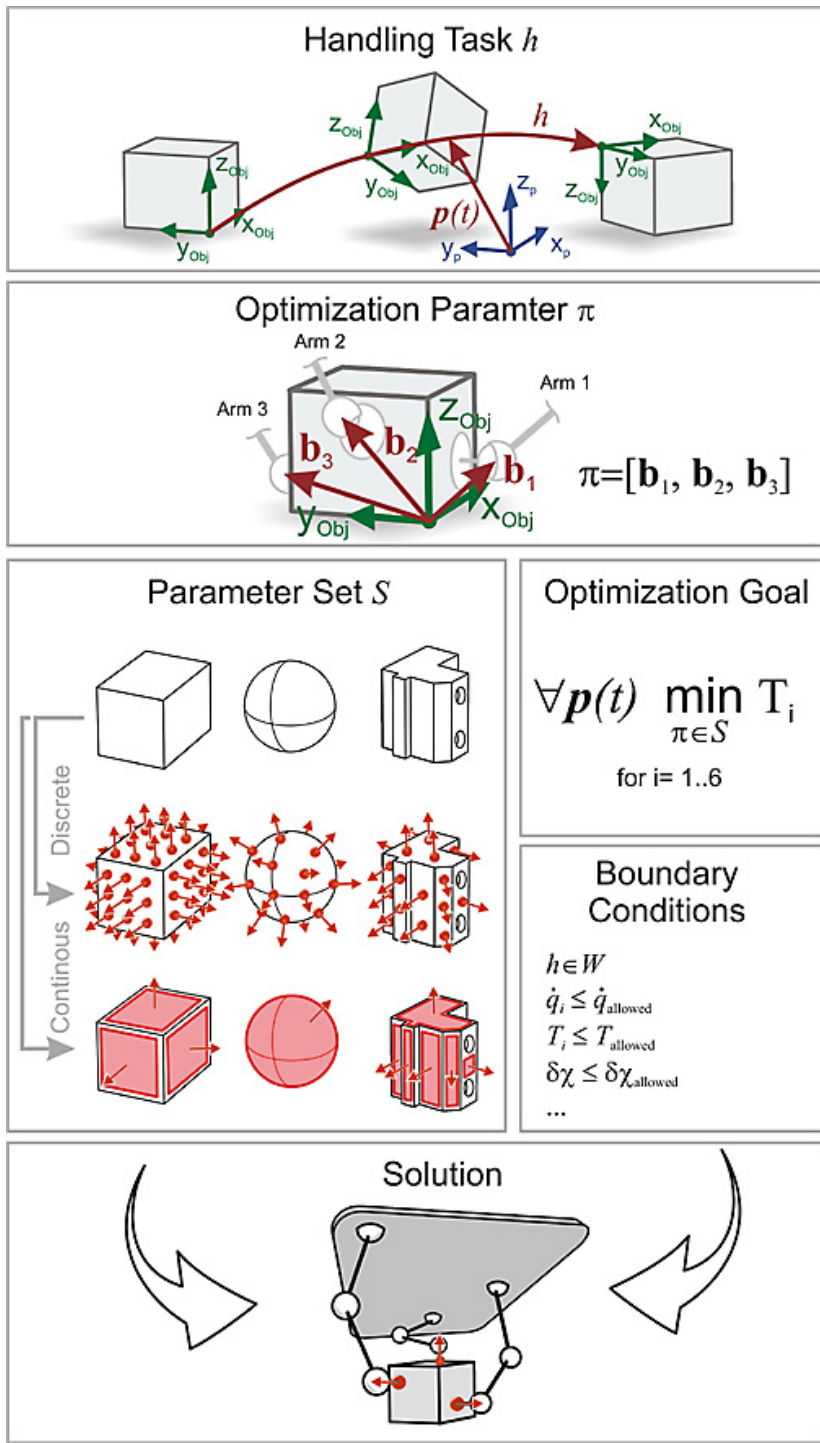

Figure 18. Grasp point optimization for e.g. minimizing drive torque.

The finding of adequate kinematic lengths on the object is similar to a dimensional synthesis of parallel manipulators. In (Angeles and Park, 2008; Nefzi et al., 2008) some optimization strategies are discussed which deal with enhancing kinetostatic performances indices all over the workspace to obtain design solutions that perform well for any trajectory.

These computations, which are used during the design process of the robot, could take several hours or even days to find one or more than one set of solutions depending on the complexity of the problem. This type of time consuming optimization for grasp planning is not acceptable as the user needs to wait after defining the handling tasks in the robot interface. The same applies to industrial applications with assembly belts featuring optical object detection via cameras, where a time critical planning of robot motion required. De- pending on the information about possible grasp areas two approaches has been investigated.

The first one deals with a given set of discrete grasp points and tries to find the best solution by browsing through these points. The second one uses the gradient-based optimization algorithm fgoalattain to search inside a set of combinations of continuous surfaces.

Both algorithms are implemented in MATLAB and integrated in a graphical user interface to control robot motion. Figure 18 shows schematically the optimization process, starting from the definition of the handling task $h$ in Workspace $\mathrm{W}$ and the parametervector $\boldsymbol{\pi}$, going on with the specification of the parameter set $\mathrm{S}$, the optimization goal and the boundary conditions, to run the optimization algorithm and find a good solution for coupling points on the object.

The optimization ensures that the chosen pointes can be grasped successfully with an underactuated wrist joint architecture by considering the combinations of the angles $\alpha_{\mathrm{R}}$ and $\alpha_{\mathrm{S}}$, as shown in Fig. 10. Furthermore collisions are observed along with the permissible range of joint motions. These restrictions guarantee that the workspace criterion is not violated. An optimization goal could be for example to find the grasp points which features the minimum actuator torque $T_{i}$ for all actuators along the path. As all kinematic performances influence each other, there is a necessity to define additional boundary conditions .These limits define $n$ maximum tolerable actuator velocities $q_{i_{-} \text {allowed }}$ and torques $T_{i \_ \text {allowed }}$, necessary object pose accuracy $\delta \chi_{\text {allowed }}$ or required stiffness $f_{\text {allowed }}$ of the robot.

To reduce computation time in contrast to general dimensional synthesis of parallel robots three simple steps are included in the grasp point optimization.

As in most cases, the handling task is explicitly given and an optimization for each object pose in the complete workspace is not target-aimed and time consuming. Hence, all performance values are only computed for the object poses, velocities and accelerations along the given path. The roughness of the path discretization determines significantly the computation time. The main time benefit arises from a pre-selection of possible grasp points or areas. Here single points or areas are checked regarding the orientations of wrist joint vector $\boldsymbol{e}_{\mathrm{WJx}}$ and surface vector $\boldsymbol{n}$ (see Fig. 5) without determining the robot performance. The reduced number of points is listed in a new set of feasible grasp combinations. An additional option to make the grasp planning even faster can be chosen by the user when the determination of adequate point is very time critical. Here the algorithm doesn't search for the best point combination, but tries to find a solution which just satisfies the given limits, as quickly as possible. 
Table 1. Results of an optimization example.

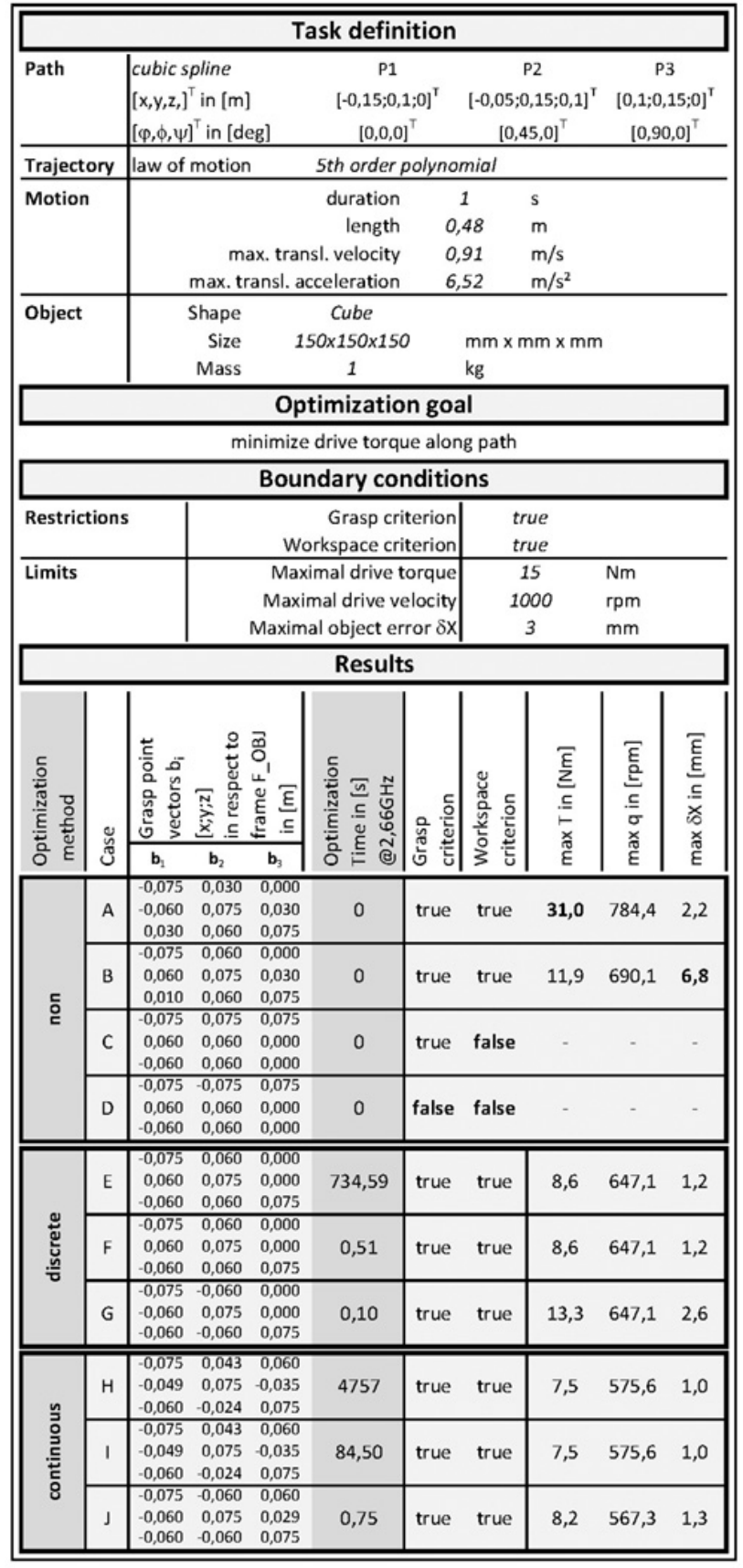

Table 1 shows the results of different optimization strategies for a given task. The first four solutions (A to D) are chosen arbitrarily and the next three (E to G) are optimized from a set of discrete start parameters and the last three solutions ( $\mathrm{H}$ to $\mathrm{J}$ ) were optimized by the basis of gradient on continuous areas.

Although the selection of user-defined grasp points takes no computation time, the choice may not satisfy all the conditions. Grasping the object in the configuration (A) causes a high maximum actuator torque during motion. Otherwise this motion is accomplished, but with a bad performance. The same applies to point combination (B), where the low accuracy of the object pose is not tolerable. However in the cases $(\mathrm{C}$ and $\mathrm{D})$ the motion is not possible at all. In $\mathrm{C}$ the object can only be picked up, but the grasp points would cause collision between the robot links and the object during motion. Furthermore the object in (D) cannot even be grasped in the initial pose. Automatic grasp planning helps the user to prevent from choosing bad points.

For cases (E to $\mathrm{G}$ ) a limited set of only 54 grasp points on the cube ( 9 per surface) is given, which can be combined theoretically in $148824(=54 ! / 51$ !) ways by the three arms. In (E) all those combinations are evaluated. This method finds the best solution in the set, but with a duration of 735 seconds which is very time consuming. In $(\mathrm{F})$ the pre-selecting process minimizes the number of points to be analyzed, which must lead to the same solution as in (E) but with a significant computing time reduction to only $0.068 \%$. Aborting the grasp planning process when the first solution is found (G) enhances the computation speed additionally to $19.6 \%$ of the total time in contrast to $(\mathrm{F})$. The solution found here is usually not the best one in the set, but still satisfies the requirements as defined in the boundary conditions.

Analogous to (E to $G$ ) the last three cases also shows one optimization without pre-selection $(\mathrm{H})$, one with preselection (I) and one with pre- and "first-best" selection (J). In these cases the parametervector $\boldsymbol{\pi}$ is optimized over continuous areas by using the gradient-based MATLAB function fgoalattain. In contrast to a discrete search these algorithms can also find solutions in between the set, which leads to better performances, however the computations takes more time. To prevent optimization from finding solutions only in local minima, each area is checked from several different start points.

In the end the user has to decide which optimization method is best for each individual case, by trading off for performance goal and computation durance.

\section{Conclusions}

In this paper, a novel approach to grasp planning was applied to the search of appropriate grasp points for a reconfigurable robot called PARAGRIP (Parallel Gripping). This new handling system is based on a parallel kinematic structure, which is generated temporarily after grasping for manipulation. As the object is integrated in the resulting closed loop mechanism, the location of the connection points are of importance for the motion performance. It was shown that these points could be carefully selected in an automatic optimization process. This grasp planning is not only focused on generating of a collision free motion with good performance but also considers the special grasp process with an underactuated arm structure. 
Acknowledgements. As parts of this work are within the scope of the excellence cluster "Integrative production technology for high-wage countries (EXC 128)", the authors thank the German Research Foundation for its support.

This project is part of the sub-category "ICD D-3.2: Flexible Assembly Systems for Selfoptimising Automation" is realized in collaboration with the Laboratory for Machine Tools and Production Engineering at RWTH Aachen University.

Edited by: J. L. Herder

Reviewed by: two anonymous referees

\section{References}

Angeles, J. and Park, F. C.: Performance Evaluation and Design Criteria, Springer Handbook of Robotics, 229-244, 2008.

Bicchi, A.: On the Closure Properties of Robotic Grasping, Int. J. Robot. Res., 14, 319-334, 1995.

Bicchi, A.: Hands for dexterous manipulation and powerful grasping: A difficult road toward simplicity, IEEE. Trans. Robot. Automat., 16, 652-662, 2000.

Birglen, L., Laliberté, T., and Gosselin, C. M.: Optimal Design of Underactuated Fingers, Underactuated Robotic Hands, Springer Berlin, ISSN 1610-7438, Vol. 40, 117-138, 2008.

Butterfass, J., Grebenstein, M., Liu, H., and Hirzinger, G.: DLR II: Next generation of a dexterous robot hand, Proc. IEEE Int. Conf. on Robotics and Automation, 109-120, 2001.

Ebert-Uphoff, I. and Gosselin, C. M.: Kinematic Study of a new Type of special parallel Platform Mechanism, DECT98/MECH5962, Proceedings of ASME Design Engineering Technical Conference, 1998.

Feldmann, K., Ziegler, C., and Michl, M.: Bewegungssteuerung für kooperierende Industrieroboter in der Montageautomatisierung, wt werkstattstechnik online, 97, 9, p. 713, 2007.

Hao, F. and Merlet, J.-P.: Multi-Criteria optimal design of parallel manipulators based on interval analysis, Mech. Mach. Theory, 40, 157-171, 2005.
Kragten, G. A. and Herder, J. L.: The ability of underactuated hands to grasp and hold objects, Mech. Mach. Theory, 45, 3, 408-425, 2010.

Krut, S.: A Force-Isotropic Underactuated Finger, Proceedings of the 2005 IEEE International Conference on Robotics and Automation, Barcelona, Spain, 2005.

Laliberté, T., Birglen, L., and Gosselin, C. M.: Underactuation in robotic grasping hands, Journal of Machine Intelligence and Robotic Control, 4, 3, 77-87, 2002.

Lee, J.-J. and Tsai, L.-W.: Structural Synthesis of Multi-Fingered Hands, J. Mech. Design, 124, 272-276, 2002.

Möller, N.: Bestimmung der Wirtschaftlichkeit wandlungsfähiger Produktionssysteme, Forschungsbericht IWB, Band 212, 2008.

Müller, R., Riedel, M., Vette, M., Corves, B., Esser, M., and Huesing, M.: Reconfigurable Self-Optimising Handling System, Precision Assembly Technologies and Systems, edited by: Ratchev, S., 5th International Precision Assembly Seminar, IPAS 2010, 1. Edn., Springer Berlin, ISBN 978-3-642-11597-4, 255262, 2010.

Nefzi, M., Riedel, M., and Corves, B.: Development and design of a multi-fingered gripper for dexterous manipulation, Mechatronic Systems, 4(1), doi:10.3182/20060912-3-DE-2911.00026, 2006.

Nefzi, M., Riedel, M., and Corves, B.: Towards an Automated and Optimal Design of Parallel Manipulators, edited by: Arreguin, J. M. R., Automation and Robotics, I-Tech, Wien, ISBN 978-3902613-41-7, 143-156, 2008.

Nyhuis, P.: Wandlungsfähige Produktionssysteme, Heute die Industrie von morgen gestalten, Garbsen, PZH, Produktionstechn. Zentrum, 2008.

Riedel, M., Nefzi, M., Hüsing, M., and Corves, B.: An adjustable gripper as a reconfigurable robot with a parallel structure, Proceedings of the Second International Workshop on Fundamental Issues and Future Research Directions for Parallel Mechanisms and Manipulators, 2008.

Yoshikawa, T. and Nagai, K.: Manipulating and Grasping forces in Manipulation by Multifingered Robot Hands, Int. J. Robotics and Automation, 7, 66-67, 1991. 